\title{
Sequence diversity in CYP3A promoters and characterization of the genetic basis of polymorphic CYP3A5 expression
}

\author{
Peter Kuehl1,2*, Jiong Zhang2,3*, Yvonne Lin ${ }^{4}$, Jatinder Lamba ${ }^{5}$, M ahfoud Assem $^{5}$, J ohn Schuetz ${ }^{5}$, Paul B. \\ Watkins $^{6}$, Ann Daly ${ }^{7}$, Steven A. Wrighton ${ }^{8}$, Stephen D. Hall ${ }^{9}$, Patrick M aurel ${ }^{10}$, M ary Relling ${ }^{5}$, Cynthia \\ Brimer $^{5}$, Kazuto Yasuda ${ }^{5}$, Raman Venkataramanan ${ }^{11}$, Stephen Strom ${ }^{12}$, Kenneth Thummel ${ }^{4}$, M ark S. Boguski ${ }^{2}$ \\ $\&$ Erin Schuetz ${ }^{5}$ \\ *These authors contributed equally to this work.
}

Variation in the CYP3A enzymes, which act in drug metabolism, influences circulating steroid levels and responses to half of all oxidatively metabolized drugs. CYP3A activity is the sum activity of the family of CYP3A genes, including CYP3A5, which is polymorphically expressed at high levels in a minority of Americans of European descent and Europeans (hereafter collectively referred to as 'Caucasians'). Only people with at least one CYP3A5* 1 allele express large amounts of CYP3A5. Our findings show that single-nucleotide polymorphisms (SNPS) in CYP3A $5 * 3$ and CYP3A5* 6 that cause alternative splicing and protein truncation result in the absence of CYP3A5 from tissues of some people. CYP3A5 was more frequently expressed in livers of African Americans $(60 \%)$ than in those of Caucasians (33\%). Because CYP3A5 represents at least $50 \%$ of the total hepatic CYP3A content in people polymorphically expressing CYP3A5, CYP3A5 may be the most important genetic contributor to interindividual and interracial differences in CYP3A-dependent drug clearance and in responses to many medicines.

\section{Introduction}

A major goal of the Human Genome Project has been completed with publication of the draft sequence of the human genome ${ }^{1,2}$. Understanding the biological and medical implications of variation in the human genome sequenceisanother major aim of the Human Genome Project ${ }^{3,4}$. Cytochromes P450 (CYP) are particularly amenable for rapid identification of functionally important sequence variations, because CYP catalytic activities can be determined by various in vivo and in vitro drug bioassays. Moreover, the applications of such information are particularly relevant for pharmacogenomics, in which knowledge of SNPs in CYP genes may lead to individualized drug dosing and improved therapeutics. Here we elucidate whether sequence variations in the promoters of genes encoding enzymes of the CYP3A family contribute to variability in CYP3A metabolism.

The cytochromes P450 evolved to catalyze the metabolism of numerous structurally diverse exogenous and endogenous molecules. Approximately 55 different CYP genes are present in the human genome and are classified into different families and subfamilies on the basis of sequence homology. The CYP families have arisen through a process of gene duplication and gene conversion. Members of the CYP3A subfamily catalyze the oxidative, peroxidative and reductive metabolism of structurally diverse endobiotics, drugs, and protoxic or procarcinogenic molecules ${ }^{6}$. The CYP3A members are the most abundant CYPs in human liver and small intestine ${ }^{7,8}$. Substantial interindividual differences in CYP3A expression, exceeding 30fold in some populations ${ }^{9}$, contribute greatly to variation in oral bioavailability and systemic clearance of CYP3A substrates, including HIV protease inhibitors, several calcium channel blockers and some cholesterol-lowering drugs. Variation in CYP3A expression is particularly important for substrates with narrow therapeutic indices, such as cancer chemotherapeutics ${ }^{10}$ and the immunosuppressants cyclosporin $\mathrm{A}$ and tacrolimus (FK506). Such variation in CYP3A can result in clinically significant differences in drug toxicities (for example, nephrotoxicity) and response (for example, graft survival). Moreover, because CYP3A metabolizes estrogens to 2-hydroxyesterone, 4hydroxyestrone and 16 $\alpha$-hydroxylated estrogens, all of which have been implicated in estrogen-mediated carcinogenicity ${ }^{11}$, variation in CYP3A may influence the circulating levels of these estrogens and the risk of breast cancer.

Human CYP3A activities reflect the heterogeneous expression of at least three CYP3A family members: CYP3A4, CYP3A5 and 
CYP3A5 gene $\stackrel{\mathrm{P} 11}{\longrightarrow}$ $1 \longdiv { 2 }$ CYP $3 A 5^{*} 1$

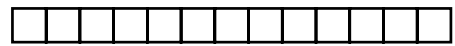
CYP $3 A 5^{*} 3$

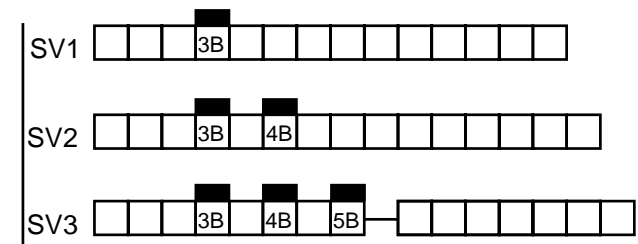

CYP $3 A 5^{*} 6$

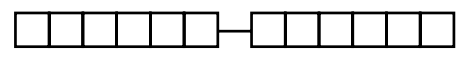

b

CYP $3 A 5^{*} 1$ CYP $3 A 5^{*} 6$

CYP3A5:

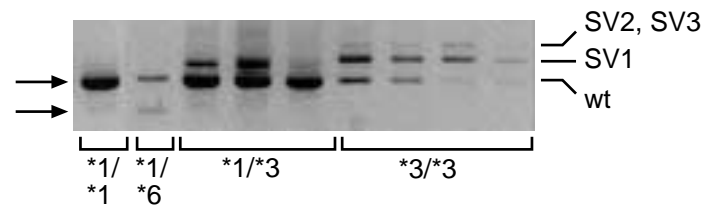

Fig. 1 Splice variants of CYP3A5. a, Schematic drawing of the cDNA structures of the various CYP3A5 alleles. Exons are numbered and boxed, and primers P11 (exon 2/3) and P6 (exon 8) are indicated. The location of intronic sequences coding for exons $3 B, 4 B$ and $5 B$ derived from intron sequences are indicated as filled boxes. b, RT-PCR analysis of mRNAs encoded for by the various CYP3A5 alleles. Total mRNA from human livers was reverse transcribed and amplified using PCR primers P11 (exon 2/3) and P6 (exon 8) and the products analyzed on agarose gels. CYP3A5 cDNA was amplified by 30 and 35 cycles of RT-PCR from hepatic RNA of people expressing high and low amounts of CYP3A5 protein, respectively. The PCR product of CYP3A5*1 is the expected size of $539 \mathrm{bp}$; the CYP3A5* 6 mRNA lacking exon 7 is $391 \mathrm{bp}$; the normally spliced CYP3A5*3 mRNA (539 bp) (wt) and the CYP3A5*3 SV1 mRNA containing exon 3B (670 bp) and SV2 mRNA containing exon $3 B$ and $4 B$ (775 bp) and the SV3 mRNA containing exons $3 B, 4 B$ and $5 B$ and missing exon 6 (769 bp). The CYP3A5*1/*1, CYP3A $5 * 1 / * 3$ and CYP3A 5*3/*3 genotypes (AA, AG and GG, respectively, at nt 22,893 in CYP3A5 (AC005020)).
CYP3A7. The CYP3A genes are adjacent to each other on chromosome band $7 q 21$, but the genes are differentially regulated ${ }^{12}$. Two CYP3A pseudogenes, CYP3AP1 and CYP3AP2, were recently identified ${ }^{12}$. Functional CYP3A4 is found in most adults, with 10to 40 -fold variation in its expression. CYP3A7 is predominantly expressed in fetal life, and its expression seems to be silenced shortly after birth; however, some people express CYP3A 7 mRNA into adulthood ${ }^{13}$. CYP3A4 activity is correlated with CYP3A4 mRNA concentrations, indicating that transcriptional control is the primary mechanism for regulating expression of CYP3A4. CYP3A5 was previously detected in livers and small intestines of some adult Caucasians, but the basis for this 'polymorphic' expression is unknown ${ }^{14-16}$. Recently, two mutations in what was believed to be the CYP3A5 promoter were found to be associated with polymorphic CYP3A5 expression ${ }^{17}$. Sequencing of the CYP3A locus, however, revealed that theseSN Ps are actually in the promoter of the pseudogene CYP3AP1 (ref. 12) and, thus, cannot be the basis for polymorphic CYP3A5 expression. The CYP3A4, CYP3A5 and CYP3A 7 promoters contain multiple putative transcription-factor-binding sites. In particular, a recently discovered nuclear receptor, PXR/SXR (for pregnane X-receptor/steroid and xenbiotic receptor), that binds to a PXR response element in the CYP3A4 and CYP3A7 promoters is important for regulation of the CYP3A proteins ${ }^{18,19}$. Therefore, we hypothesized that polymorphisms affecting CYP3A activity are present in DNA regulatory sequences. Through a systematic analysis of genomic DNA from a large number of patients with various drug metabolism phenotypes, and through analysis of DNA from the Coriell DNA Polymorphism Discovery Resource (DPDR; ref. 3), we identified all common variants in the promoter regions of CYP3A4, CYP3A5, CYP3AP1 and CYP3A 7, and demonstrated ethnic differences in allele frequencies. By performing quantitative immunoblotting and in vitro kinetic assays of drug metabolism, we evaluated the relationship between these common genetic variants and ethnic- and tissue-specific phenotypic variability. In addition, analysis of human liver CYP3A5 CDNA revealed that only those people with a CYP $3 A 5^{*} 1$ allele produce high levels of full-length CYP3A5 mRNA and express CYP3A5. Those with the CYP3A $5 * 3$ allele have sequence variability in intron 3 that creates a cryptic splice site and results in the generation of CYP3A5 exon $3 B$; this CYP3A5*3 allele encodes an aberrantly spliced mRNA with a premature stop codon. This finding explains the molecular defect responsible for one of the most common polymorphisms in drug-metabolizing enzymes.

\section{Results}

We initially performed SNP analysis of the promoter regions of the CYP3A genes on DNA from the smallest DPDR subset (Table 1). The minimum DPDR panel contained five variant alleles with a frequency greater than or equal to $1 \%$ in the combined study population. These variant alleles included the CYP3A4*1B allele, in which an $A \rightarrow G$ variant at nt -288 is present in the nifedipinespecific element of the promoter ${ }^{20}$. We detected the CYP3AP $1 * 1$ reference allele ( $\mathrm{G}$ at $\mathrm{nt}-44$ in the promoter) in 43 people ( 28 heterozygotes and 15 homozygotes). In an unusual haplotype, hereafter referred to as CYP $3 A 7^{*} 1 \mathrm{C}$ (Table 1 ), an approximately 60-bp stretch (nt - 129 to -188) of the CYP3A 7 promoter seems to have been replaced with sequence identical to the same region in the CYP3A4 promoter. This replacement indicates the occurrence of a gene conversion event ${ }^{21}$.

The Coriell DPDR panel was designed to represent the genetic diversity of United States residents who have ancestors from Europe, Africa, the Americas and Asia. We found no variants in the DPDR panel that were not also seen in the combined study population, but additional CYP3A alleles found in our larger combined population were not present in the DPDR. Most of these alleles were rare (that is, they were seen in only one or two people). To assess the usefulness of the DPDR panel in predicting SN Ps, we calculated the probability that common variants would be missed in a random sampling of our combined population. The chance of missing the polymorphic alleles CYP3A4*1B and CYP3AP $1 * 1$ was less than $5 \%$, even if the sample consisted of as few as 10 study subjects from the combined population group. If we were to randomly select a sample of 25 subjects, the probability 


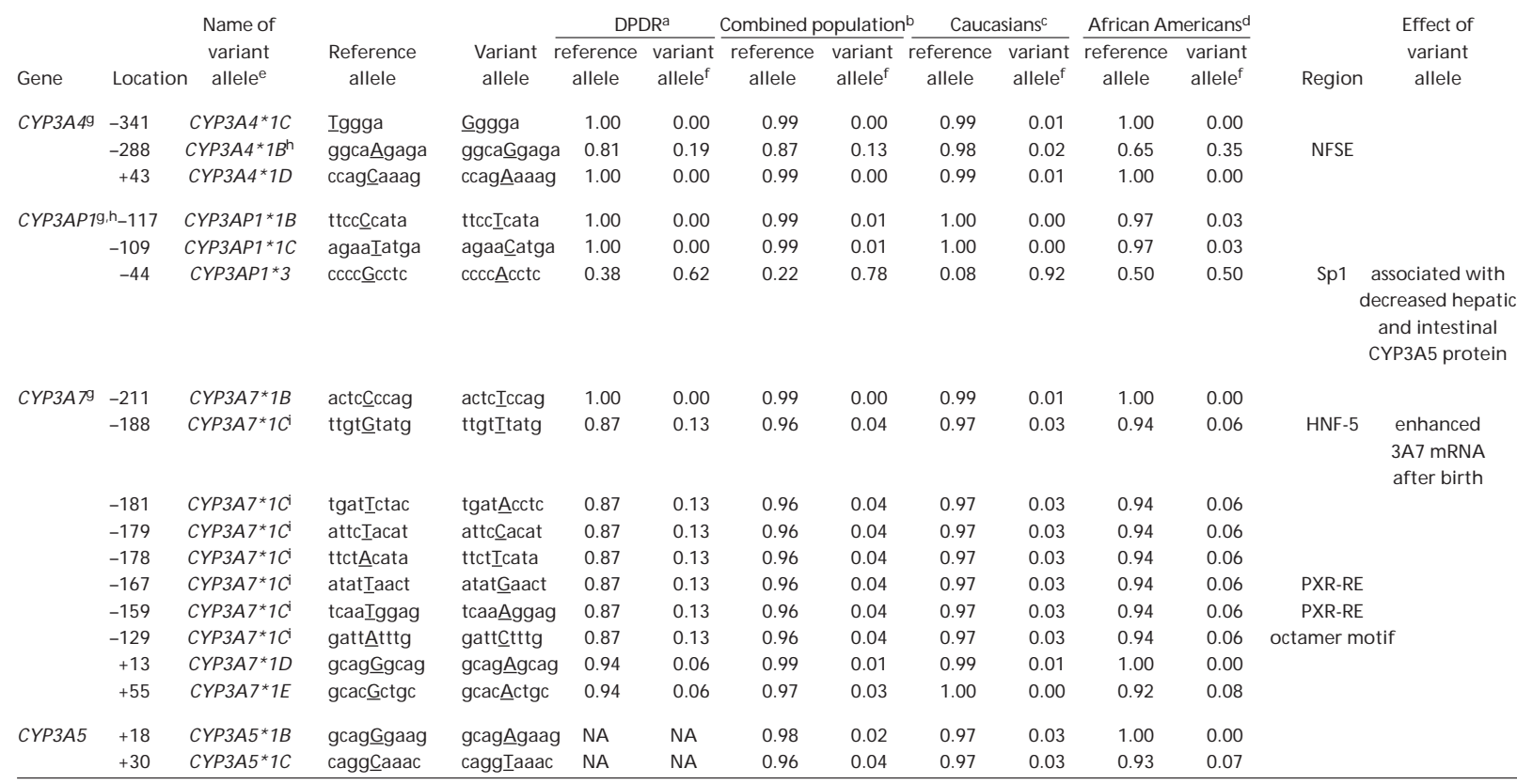

aAnalyzed people=8. ${ }^{b}$ Analyzed people analyzed people=119 (CYP3A4/7), 123 (CYP3A5P1) or 74 (CYP3A5). cAnalyzed people=79 (CYP3A4/7), 83 (CYP3A5P1) or 59 (CYP3A5). ${ }^{d}$ Analyzed people $=40$ (CYP3A4/7/P1) or 15 (CYP3A5). ${ }^{2}$ The CYP alleles are named according to recommended nomenclature guidelines ${ }^{44}$ (http://www.imm.ki.se/CYPalleles). f The frequency of the variant alleles was calculated by using the formula: frequency $=$ [ $2 *$ (number of people homozygous for the variant allele)+(the number of heterozygous people)]/[2*(total number of people)]. The frequency of the reference allele was equal to the following: 1-(variant allele frequency). For DNA from families, we included only data gathered from the parents' samples in the frequency calculations. 9The CYP3A4*1, CYP3A $7 * 1$ and CYP3A5*1 reference alleles are D11131, AF280107 and AC005020, respectively. Apparent errors in the original sequence of the CYP3AP1 promoter are corrected and deposited as the consensus sequence for CYP3AP1*1. The variants are numbered relative to the initiation site of transcription, which is defined as +1 . To renumber alleles according to the translation start site (A in initiation codon ATG is +1 ), subtract 103 bases from the SNP location (for example, the location of CYP3AP1*3 changes from -44 to -147 ). ${ }^{\text {h } C Y P 3 A 4 * 1 B ~ a n d ~ C Y P 3 A P 1 * ~} 1$ are partially linked in African Americans. 'These seven CYP3A7 variants (hereafter referred to as CYP3A $7 * 1 C$ ) are located between $n t-129$ and -188 and are in complete linkage.

of missing polymorphic CYP $3 A 7 * 1 \mathrm{C}$ alleles would be $15 \%$, that of missing CYP3A $7 * 1 E$ would be $25 \%$, and that of missing CYP3A $7 * 1 \mathrm{D}$ would be greater than $60 \%$. The probability of missing the rare alleles CYP3A $4 * 1 C$, CYP3A4*1D, CYP3AP $1 * 1 B$ and CYP3AP1*1C would begreater than $60 \%$. ThustheDPDR 8-sample subset was more useful in accurately predicting the identity of CYP3A SN Ps than a random sampling of 25 peoplefrom the combined population.

As has been previously reported 22, CYP3A4*1B occurred at a much higher frequency in African Americans $(q=0.35)$ than in Caucasians ( $\left.q=0.02 ; \chi^{2}=48.9, p<0.001\right)$. We found no SN Ps in the regions containing the proximal (nt -169 to -152 ) and distal (nt - 7836 to - 7208; ref. 23) PXREs of CYP3A4. Sequence analysis of the CYP3A5 promoter (nt +90 to -280 ) revealed two SNPs in the $5^{\prime}$ UTR (Table 1). CYP $3 A 7 * 1 C$ wasthreetimes morecommon in an African American population $(q=0.06)$ than in a Caucasian population ( $q=0.03$ ). Among the 10 unrelated French Caucasians in the study, however, 3 people were heterozygous for CYP3A $7 * 1 C(q=0.15)$, whereas only 2 of the 96 unrelated nonFrench Caucasians carried 1 CYP3A $7 * 1 C$ allele $(q=0.01)$. CYP3AP $1 * 1$ was the most common minor allele in Caucasians and African Americans. This allele is also divergently distributed among African Americans ( $q=0.50)$ and Caucasians ( $q=0.08$, $\left.\chi^{2}=51.1, P<0.001\right)$. By examining other panels of $D N A$, we found that the frequency of CYP3AP $1 * 1$ alleles ( $q$ values) in Japanese was 0.15; in Chinese, 0.3; in M exicans, 0.13; in Southeast Asians (excluding Japanese and Chinese), 0.5; in Pacific Islanders, 0.3; and in Southwestern American Indians, 0.5 .

We determined the association of CYP3A alleles with CYP3A phenotypes. None of the SNPs in the CYP3A5 or CYP3A4 promoters, including the CYP3A4*1B allele, were associated with altered CYP3A activities of people whose phenotypes had been determined in vivo by the erythromycin breath test or nifedipine clearance or in vitro by assays for total midazolam hydroxylase or for basal or rifampin-inducible testosterone $6 \beta$-hydroxylase activity. Previous studies have noted the polymorphic expression of CYP3A5 in the liver and intestine of approximately $25 \%$ of people studied ${ }^{14,16,24}$. Although there is good concordance of CYP3AP1*1 ( $G$ at nt -44) with high levels of CYP3A 5 in the liver ${ }^{17}$, CYP3AP $1 * 1$ cannot be responsible for polymorphic CYP3A5 expression. Because we did not find a CYP $3 A 5$ promoter variant that correlated with CYP3A5 expression, we determined whether CYP3AP $1 * 1$ was in linkage disequilibrium with another variant in CYP3A5 that might be causal. Because the CYP3AP1 genotype is correlated with CYP3A5 expression ${ }^{17}$, we used mRNAs from Caucasian and African American livers representing the CYP3AP $1 * 1 / * 1$, CYP3AP $1 * 1 / 33$ and CYP3AP $1 * 3 / * 3$ genotypes to generate and sequence the entire CYP3A5 CDNA. The CYP3A5 mRNA content was greater in people with a CYP3AP1*1 allele than in those people homozygous for CYP3AP1*3. All livers contained the full-length CYP3A5 mRNA transcript encoded by CYP3A5*1 (Fig. 1). M any peoplecarrying CYP3AP $1 * 3$ also carried CYP3A $5 * 3$, which encodes only a small amount of normally spliced CYP3A5 mRNA and a splice variant-1 (SV1) mRNA. SV 1 results from a SN P at nt 22,893 (in AC005020), which creates a cryptic consensus splice site and exon 3B, an exon derived from intron-3 sequences of CYP3A5 (Fig. 1 and Table2). The mutated sequence in intron 3 of CYP3A $5 * 3$ has a splicesite prediction score of 0.9 . Because of the prematuretermination codon in exon 3B, the SV 1 mRNA encodes a protein whose sequence is truncated after amino acid 102 . We found a second 


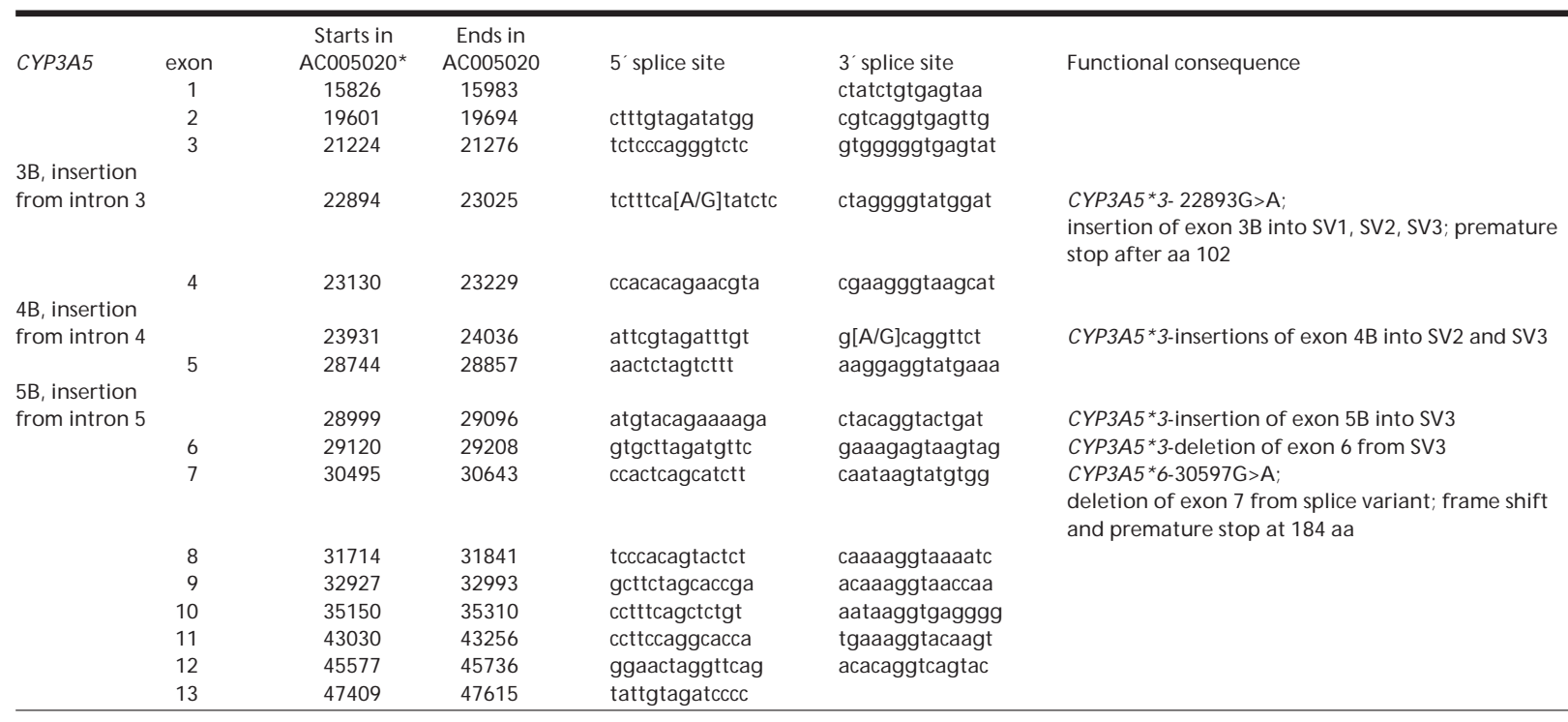

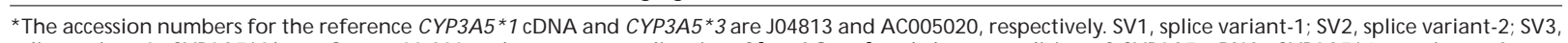

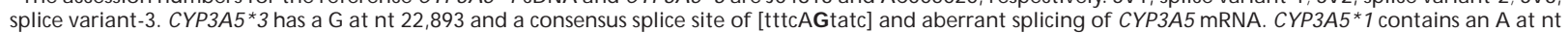

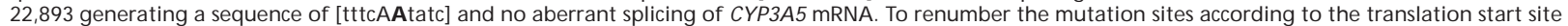
(nt 15913 is A in the ATG initiation codon), subtract 15912 bases from the SNP location (for example, the location of CYP3A5*6 is $14685 G>A$ ).

splice variant (SV2) mRNA that encoded exons 3B and 4B in many people carrying CYP $3 A 5 * 3$. Although sequenceanalysis around the predicted splice site for exon 4B revealed a SNP at nt 24,035 (Table 2), this SNP and the generation of SV2 werenot related (Table2). A third splice variant (SV3) mRNA comigrated with SV2 in an agarose gel (Fig. 1 ) and contained exons $3 B, 4 B$ and $5 B$, but not exon 6. No SN Ps were found around exon $5 B$ or exon 6 that correlated with the SV 3 transcript. The fact that mRNAs with premature stop codons are more unstable and rapidly degraded ${ }^{25}$ may explain the lower amount of CYP3A5 mRNA in people homozygous for CYP3A 5*3 compared with people carrying at least one CYP3A5*1 allele. We examined the frequency of the CYP3A5*1 allele and found it to be distributed divergently among African Americans $(q=0.45)$ and Caucasians $(q=0.15)$. By examining other panels of DNA, wefound that the frequency of CYP3A $5 * 1$ alleles (q values) in theDPDR subset of 8 peoplewas 0.31 ; in Japanese, 0.15 ; in Chinese, 0.35; in M exicans, 0.25 ; in Southeast Asians (excluding Japanese and Chinese), 0.33; in Pacific I slanders, 0.35; and in Southwestern American Indians, 0.6 . CYP3A5*6 was identified in only 3 of 20 African Americans and encoded a normal CYP3A5 mRNA and a splice variant mRNA in which exon 7 was deleted. This deletion resulted in a frameshift, causing the encoded protein to be truncated at amino acid 184. Sequence analysis of genomic DNA and cDNA synthesized from the spliced variant mRNA from thoseindividuals skipping exon 7 identified a single sequence variation: a $30597 \mathrm{G}>\mathrm{A}$ synonymous mutation in exon 7 (nt $711 \mathrm{G}>\mathrm{A}$ in the CYP3A5 CDNA). This genotype was concordant with the loss of exon 7. This silent mutation may cause exon 7 skipping by disrupting an exonic splicing enhancer ${ }^{26}$.

To determine the association of the CYP3A5 and CYP3AP alleles with CYP3A5 expression, we measured CYP3A5 protein concentration in liver specimens. We found a greater frequency of CYP3A5 ( $\geq 21 \mathrm{pmol} / \mathrm{mg}$ protein) in African Americans (11/20; $50 \%$ ) compared with Caucasians (9/27; 33\%). All Caucasians and most African Americans with a CYP3A 5 content less than 21 $\mathrm{pmol} / \mathrm{mg}$ protein were homozygous for the CYP $3 A 5 * 3$ allele with $G$ at $n t 22,893$ leading to cryptic CYP3A5 mRNA splicing, whereas those with CYP3A5 content ranging from 21 to 202 $\mathrm{pmol} / \mathrm{mg}$ had at least one CYP3A5*1 allele (A at $\mathrm{nt} 22,893$ with no aberrant CYP3A 5 mRNA splicing; Fig. 2a,b). Among thelivers of Caucasians, the CYP3A5 allele frequencies conformed to $\mathrm{H}$ ardy-Weinberg equilibrium. There was al so complete concordance between the CYP3A $5 * 1$ and CYP3AP $1 * 1$ genotypes and between CYP3A5*3 and CYP3AP1*3 genotypes in Caucasians. Among African Americans, however, there were several outliers when the CYP3AP1 genotype was used to predict African American CYP3A5 phenotype. Specifically, African American liver 788 had a relatively high level of CYP3A5 ( $54 \mathrm{pmol} / \mathrm{mg}$ protein) that was not predicted by a CYP3AP1*3/*3 genotype, but was explained by theCYP3A $5 * 1 / * 3$ genotype. African American livers 624 and 958.29 had relatively low levels of CY $3 A 5$ protein that were not predicted by a CYP3AP $1 * 1 / * 1$ gen otype, but were better explained by CYP3A $5 * 1 / * 6$ genotypes because the CYP3A $5 * 6$ allele generates an mRNA lacking exon 7. The final outlier (African American liver 50) had only a trace amount of CYP3A5 protein that was not predicted by the CYP3AP $1 * 1 / * 3$ genotype or the CYP $3 A 5 * 1 / * 3$ genotype. This discrepancy may be due to secondary degradation of CYP3A5 and other proteins following poor tissue preservation, or to a second mutation in the coding region of CYP3A5 that confers instability of the protein (for example, CYP3A5*2; ref. 27).

We examined the rates of midazolam hydroxylation by the same 47 livers. CYP3A4 and CYP3A5 each produce two metabolites: 1'-hydroxymidazolam (1'-OH MDZ) and 4-hydroxymidazolam (4-OH M DZ). The mean reaction velocities were 2.5 -fold $(P=0.03)$ higher for the livers of Caucasians and 2.2-fold $(P=0.19)$ higher for the livers of African Americans with at least one CYP $3 A 5^{*} 1$ allele, compared with those livers of people who were homozygous for CYP3A5*3 (Fig. 3). The ratio of $1^{\prime}-\mathrm{OH}$ MDZ to 4-OH MDZ is dependent on substrate concentration and the type of CYP3A enzyme ${ }^{28}$. At a midazolam concentration of $8 \mu \mathrm{M}$, the ratio was 5.5 when we used recombinant CYP3A4 and 16.1 when we used recombinant CYP3A5. Thus, with liver microsomes, the observed product ratio depends on the relative amounts of CYP3A4 and CYP3A5 protein. The average ratio of $1^{\prime}-\mathrm{OH}$ MDZ to 4-OH M DZ was higher in Caucasian livers with at least one CYP $3 A 5^{*} 1$ allele $(8.1 \pm 3.0$ for $* 1 / * 3$ genotype) than in thosewith two CYP3A $5 * 3$ alleles $(5.9 \pm 1.7$ for $* 3 / * 3 ; P=0.09)$. The 
Fig. 2 Relationship between CYP3A5 phenotype and CYP3A5 genotype in livers of Caucasians (a) and African Americans (b). Microsomal content of CYP3A5 protein in human livers (HL) was determined by immunoblot analysis. Identification of the CYP3AP1 and CYP3A5 genotypes were made by direct sequencing of genomic DNA: the CYP3API*1* $1 *$, CYP3AP1*1/3 3 and CYP3AP1*3/*3 genotypes (GG, AG and $A A$, respectively at $n t-44$ of the CYP3AP1 promoter). The CYP3A5 genotypes are defined in Fig. 1.

average ratio of $1^{\prime}-\mathrm{OH} M D Z$ to $4-\mathrm{OH} M D Z$ was higher in African American livers with at least one CYP3A $5 * 1$ allele $(9.8 \pm 3.8$ for $* 1 / * 1, * 3, * 6$ genotypes) than in those with two CYP3A5*3 alleles $(8.3 \pm 1.6$ for $* 3 / * 3 ; P=0.41$ ).

M easurement of CYP3A quantity in the same 47 livers showed that CYP3A4 content was not significantly different between Caucasian and African American livers. Within each ethnicity there was no difference in mean CYP3A4 content between livers with or without at least one CYP3A5*1 allele. The amount of total CYP3A in Caucasian and African Americans was approximately threefold higher in those with the CYP3A5*1 allele than in people not carrying this allele ( $P=0.001$ and $P=0.01$, respectively). Moreover, CYP3A5 protein represented more than $50 \%$ of the total CYP3A content in onethird of Caucasian livers and over one-half of African American livers. Thus, CYP3A5 represented a considerably greater proportion of total CYP3A than previously estimated ${ }^{14}$ and contributed significantly to CY P3A content and catalytic activity.

Because CYP3A5 is variably expressed in human intestine $e^{16}$, we took advantage of a unique cohort of paired samples of small intestine and liver to determine whether the CYP3AP1*1 genotype-phenotype concordance is seen in extrahepatic tissues. All people with large quantities of CYP3A5 in the liver also had relatively high intestinal levels of CYP3A5 and were heterozygous or homozygous for the CYP3A5*1 allele (Fig. 4). Further, the intestinal midazolam product ratio for people with at least one CYP $3 A 5 * 1$ allele ( $9.6 \pm 2.5$ for the AG genotype) differed from that for people with two CYP3A5*3 alleles $(6.0 \pm 0.3 ; P=0.06)$.

The CYP $3 A 4 * 1 B$ allele was originally proposed to beassociated with altered CY P3A4 hepatic activity20,29,30, but this proposal has been controversial 31,32. Because the CYP3A4*1B and CYP3AP1*1 or CYP3A4*1B and CYP3A5*1 alleles can all be present in the same person, we hypothesized that it is ultimately the CYP3A5 genotype that influences the overall functional activity of CYP3A. African Americans frequently carried both CYP3AP1*1 and CYP3A4*1B alleles (Table 3), and using data from those people homozygous for CYP3A 4*1B or CYP3AP $1 * 1$, we determined the linkage of the two alleles (data not shown; $\chi^{2}=12.8, P<0.001$ ). The association of the CYP3A $5 * 1$ and CYP $3 A 4 * 1 B$ alleles was not significant in African Americans, but approached statistical significance in Caucasians.

The CYP3A 7 promoter contains a set of 7 tightly linked variants that replaced $60 \mathrm{bp}$ of the CYP3A7 promoter with the identical region from the CYP3A4 promoter; thus this replacement altered 3 transcription-factor-binding sites ${ }^{33}$ : hepatic nuclear factor-5 (H NF-5), an octamer motif and the PXRE. Although CYP3A7 is generally expressed only during fetal development,
CYP3A7 mRNA is detected in a small fraction of adults ${ }^{13}$. We speculated that these nucleotides are important for the increased expression of hepatic CYP3A4 and loss of CYP3A 7 expression in most people after birth. We evaluated hepatic CYP3A7 mRNA expression in nine adult livers (five with the variant CYP3A 7*1C allele) to determine whether this replacement of CYP3A7 promoter elements by CYP3A 4 sequences is related to expression of CYP3A7. The two people with the highest levels of CYP3A7 mRNA carried CYP3A7*1C, and four of five people with CYP3A 7*1C alleles expressed CYP3A7 mRNA (data not shown). One person with the CYP3A $7 * 1 C$ allele had no CYP3A7 mRNA, however, and two people lacking CYP3A $7 * 1 C$ had very low but detectable levels of CYP3A 7 mRNA. Thus, CYP3A7*1C is associated with CYP3A7 mRNA expression, but is not the sole explanation for CYP3A 7 expression in adults.

\section{Discussion}

Our findings show that SN Ps in CYP3A5 that cause alternative splicing and truncation of CYP3A5 protein provide a molecular explanation for the absence of CYP3A5 protein from some people. The most common cause of the loss of hepatic CYP3A5 

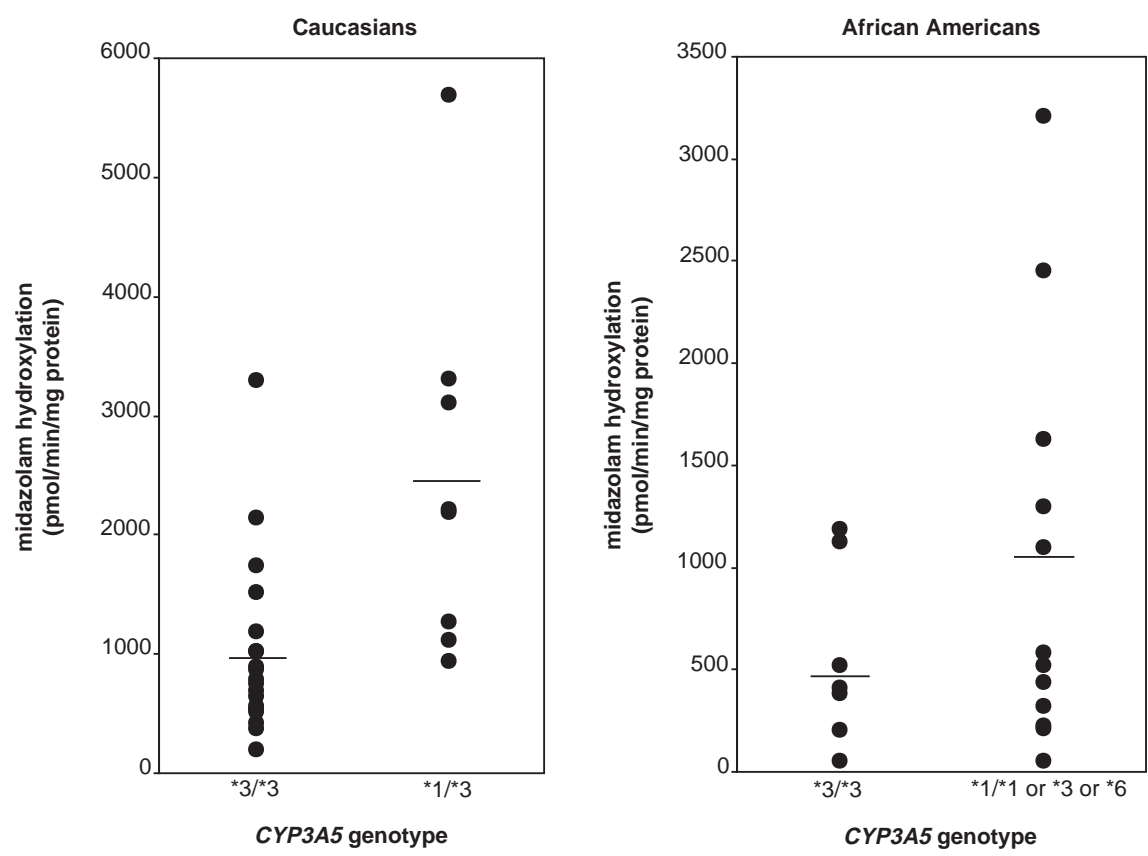

Fig. 3 Rates of midazolam hydroxylation in livers with different CYP3A5 genotypes. Total rate of midazolam hydroxylation in liver microsomes was measured. The CYP3A5 genotypes are defined in Figs. 1 and 2. Bars indicate mean values for the subpopulations.

inhibited by some compounds, polymorphic CYP3A5 expression will contribute to differences in metabolite profiles and in susceptibility to inhibitory drug interactions. One can also envision an increased risk of adverse effects from CYP3A5generated toxic metabolites in people that predominantly express CYP3A5. For instance, the rate of $3 \alpha$-hydroxylation of aflatoxin $B_{1}$ (the inactivation step) by CYP3A5 is approximately $1 \%$ of that of CYP3A4, whereas CYP3A5 efficiently catalyzes the 8,9-epoxidation (the activation step) of aflatoxin $B_{1}$ (ref. 36). Thus, in those for expression is a SNP at nt 22,893 (AC005020) in intron 3 of CYP3A5*3; this SN P generates a cryptic splice site and exon 3B. Splicing of this exon into the CYP3A $5 * 3$ transcript introduces a stop codon. Thus, translation of the abnormal transcript generates a protein that is prematurely terminated at amino acid 109. The new CYP3A5*6 allele, which was identified in African Americans, contained a 30597G >A mutation in exon 7. This SNP correlates with the deletion of exon 7 from the CYP3A5 mRNA and lower CYP3A5 catalytic activity in three African Americans. CYP3A5 expression was also closely associated with the CYP3AP1*1 genotype ${ }^{12,17}$; however, the CYP3A5 phenotype was better predicted by CYP3A5 genotype. Comparison of CYP $3 A P 1 * 1$ and CYP $3 A 5 * 1$ genotypes among the DPDR subset revealed only $37.5 \%$ linkage; in M exicans, only $65 \%$ linkage; and in Japanese, Southeast Asians, Chinese and Pacific Islanders, 90-95\% linkage. Although we detected small amounts of normally spliced CYP3A5 mRNA and resulting protein even in those people homozygous for CYP $3 A 5 * 3$, cryptic splices sites within intron 3 were used preferentially. Thus, only those people with at least one CYP3A $5 * 1$ allele polymorphically express high amounts of CYP3A5. These results add to the growing list of splicing alterations that affect the expression of clinically important genes ${ }^{34}$.

In addition to determining the basis for polymorphic CYP3A5 expression, we demonstrated that in people with at least one CYP3A5*1 allele, the peak hepatic content of CYP3A5 is much higher than previously thought ${ }^{24,35}$ and is approximately the same as the reported peak level of CYP3A4 (ref. 8). For most Caucasians and African Americans who carry the CYP3A5*1 allele, we also found that CYP3A5 accounts for at least $50 \%$ of the total CYP3A content. Thus, CYP3A5 should contribute substantially to thetotal metabolic clearance of the many CYP3A substrates. Indeed, we predict that people with the highest clearance and lowest oral bioavailability of CYP3A substrates will be heterozygous or homozygous for CYP3A5*1. These people might be more likely to encounter a lack of efficacy from a standard dose of active parent drug. Moreover, because CYP3A4 and CYP3A5 have varying degrees of catalytic capability and regioselectivity toward some substrates and because CYP3A4 and CYP3A5 are differentially whom CYP3A5 is the dominant hepatic CYP3A, the relativelack of aflatoxin $B_{1}$ detoxification compared to activation of this agent might enhance the genotoxicity of aflatoxin ${ }^{36}$. Because polymorphic CYP3A5 is one factor contributing to individual variation in CYP3A-mediated metabolism of drugs ${ }^{14,35}$, simple DNA-based tests can now be used to determine how individual differences in CYP3A5 contribute to the overall metabolic fate of these CYP3A substrates, to their pharmacodynamic variability and to diseaserisk.

CYP3A5 is the primary CYP3A family member expressed outside the liver and intestine (for example, kidney, lung and polymorphonuclear leukocytes), indicating that CYP3A5 has an important physiologic function in these tissues. Indeed, CYP3A5 can mediate the metabolism of cortisol to $6 \beta$-hydroxycortisol, a physiologic regulator of $\mathrm{Na}^{+}$transport in renal epithelia ${ }^{37}$. Variable and polymorphic renal expression of CYP3A5 (refs. 38,39) may contribute to individual differences in the localized generation of $6 \beta$-hydroxycortisol within the nephron and may have an etiologic role in, for example, salt-sensitive hypertension by increasing renal retention of $\mathrm{Na}^{+}$. Thus, CYP3A 5 is not just a catalyst of drug detoxification, but, in organs such as the kidney, it may serve an important function in regulating the pool of endogenous paracrine or endocrine factors. Similarly, polymorphic expression of CYP3A5 may contribute to variable metabolism of steroids in the prostate and breast, to differences in the concentrations of circulating steroids and, hence, to risk of disease in these tissues.

Among the Caucasians and African Americans studied here, the CYP3A5*1 genotype was associated with significant levels of CYP3A5 protein in theliver and small intestine, thetwo main tissues in which CYP3A5 is likely to makethe greatest contribution to drug elimination. The polymorphic distribution of the CYP $3 A 5^{*} 1$ allele indicates that relatively high levels of metabolically active CYP3A5 are expressed by an estimated $30 \%$ of Caucasians, 30\% of Japanese, $30 \%$ of M exicans, $40 \%$ of Chinese and more than $50 \%$ of African Americans, Southeast Asians, Pacific Islanders and Southwestern American Indians. The higher prevalence of CYP3A5 expression indicates that these non-Caucasians are more likely to experience higher clearance of drugs principally inactivated by CYP3A; are less 
Fig. 4 Association between CYP3A5 expression and CYP3A5 genotype in human liver and small intestine. Eleven pairs of tissue obtained from the same donors (all Caucasian) were analyzed for CYP3A5 protein content; the CYP3AP1 and CYP3A5 genotypes are defined in Figs. 1 and 2. CYP3A5 content ranged from undetectable levels to $31 \mathrm{pmol} / \mathrm{mg}$ of intestinal homogenate protein. NA, not available. Intestine ratio $=\mathrm{I}^{\prime} \mathrm{-OH} \mathrm{MDZ}$ formation $(\mathrm{pmol} / \mathrm{min} / \mathrm{mg}) / 4-\mathrm{OH} \quad \mathrm{MDZ}$ formation ( $\mathrm{pmol} / \mathrm{min} / \mathrm{mg})$. Low activity, less than 25 pmol 1'-OH MDZ per min per $\mathrm{mg}$ homogenate protein (limit of quantitation).

likely to experience dose limiting toxicities; and have different risks of diseases that are associated with the CYP3A5 expressor phenotype. Natural selection could drive the high frequency of CYP3A5*1 allelesin African Americans and equatorial ethnic groups if, for example, there was a causal link between CYP3A5 expression and renal metabolism of endoge nous molecules that affect $\mathrm{Na}^{+}$retention ${ }^{37}$. Such a link might confer a selective advantage in areas of water shortage.

Using the CYP3A 4*1B allele as a marker, several investigators have postulated that CYP3A4 is a candidate gene in the development of several disorders including prostate cancer and epipodophyllotoxin-induced secondary acute myelogenous leukemia20,29,30. Our finding of the simultaneous occurrence of CYP3A4*1B, CYP3AP $1 * 1$ and CYP3A $5 * 1$ alleles in some people indicates the need to re-evaluate this association and to determine whether the CYP3A 5 genotype and CYP3A 5 expression are more closely associated with these disease risks.

Data on the frequencies of SNPs in human genes have been largely gathered from surveys of exons and introns ${ }^{40,41}$, with little specific attention given to the frequency of SN Psin upstream regulatory regions, particularly those of differentially regulated gene family members. Previous studies have reported a nucleotide diversity of about 1 in 2,000 bp of noncoding DNA (consisting mostly of $5^{\prime}$ and $3^{\prime}$ UTRs and introns) and lower frequencies in coding sequences; the least frequent SN $\mathrm{P}$, with a nucleotide diversity of approximately 1 in 20,000 bp, was a coding SN P that causes a nonconservative amino acid substitution ${ }^{41}$. In Caucasians, the observed nucleotide diversity in the CYP3A4 promoter was 1 in 7,246 bp; in the CYP3AP 1 promoter, 1 in 2,577 bp; in the CYP3A 5 promoter, 1 in 3,175 bp; and in the CYP $3 A 7$ promoter, 1 in 4,444 bp. In African Americans the observed nucleotide diversity in the CYP3A4 promoter was 1 in 1,000 bp; in the CYP3AP1 promoter, 1 in $649 \mathrm{bp}$; in the CYP $3 A 5$ promoter, 1 in 2,841 bp; and in the CYP3A 7 promoter, 1 in 1,677 bp. It will be of interest to compare the level of nucleotide diversity within the CYP3A promoters with a much larger diverse survey of human gene promoters.

Traditionally, pharmacogenetics research used the strategy of identifying outliers in drug response, obtaining the DNA from outliers and identifying sequence variation in genes involved in that drug's metabolism. In the postgenomic era, reverse pharmacogenetics approaches are under way in which common

\begin{tabular}{|c|c|c|c|c|}
\hline Table $3 \cdot$ Pre & of CYP3A4* 1B & d CYP3AP1*1 or & $3 A 4 * 1 B$ and & $3 A 5^{*} 1$ in one $p$ \\
\hline Caucasians & CYPЗАР $1 * 3 / * 3$ & $\begin{array}{c}\text { At least one } \\
\text { CYP3AP1*1 allele }\end{array}$ & CYP3A5*3/*3 & $\begin{array}{c}\text { At least one } \\
\text { CYP3A5*1 allele }\end{array}$ \\
\hline $\begin{array}{l}\text { CYP3A4*1/*1 } \\
\text { CYP3A4* } 1 \mathrm{~B}\end{array}$ & $\begin{array}{r}63 \\
1\end{array}$ & $\begin{array}{r}10 \\
2\end{array}$ & $\begin{array}{r}14 \\
0\end{array}$ & $\begin{array}{l}3 \\
2\end{array}$ \\
\hline African Americ & & & & \\
\hline $\begin{array}{l}\text { CYP3A4*1/*1 } \\
\text { CYP3A4*1B }\end{array}$ & $\begin{array}{r}11 \\
3\end{array}$ & $\begin{array}{r}3 \\
15\end{array}$ & $\begin{array}{l}3 \\
2\end{array}$ & $\begin{array}{l}3 \\
9\end{array}$ \\
\hline
\end{tabular}

polymorphisms are first identified in panels of DNA from anonymous people and then screening for these common sequence variations is carried out in people whose drug metabolism phe notype is known. For genes of pharmacogenetic interest, or for any gene, the fewer the number of DNA samples required to detect these common genetic variants, the more rapidly and cost effectively common variants can be identified. Moreover, these common variants are proposed to significantly contribute to risks of common disease ${ }^{4}$. Our results confirm that the smallest subset of eight DPDR samples was sufficient to identify all common polymorphisms in the CYP3A promoters and in the gene CYP3A5, including two polymorphisms that had a high probability of being missed in a random population sample of 25 people. This result indicates the robust power of this resource for rapid SN P discovery.

\section{Methods}

Population samples. We sequenced the CYP3A promoters of 159 people. Eight samples were from the DPDR (Coriell Cell Repositories). We also obtained samples from the following sources: 47 livers (27 Caucasian donors, 20 African American donors) whose midazolam 1'-hydroxylase and 4-hydroxylase activities and CYP3A5 and CYP3A4 protein were characterized (University of Washington, University of Pittsburgh, the M edical College of Virginia and St. Jude Children's Research Hospital); primary human hepatocytes from 8 people whose basal and drug-inducible testosterone $6 \beta$-hydroxylase activity had been characterized (University of Pittsburgh); kidneys from 12 people (Indiana University); 29 subjects (representatives of 7 families) whose nifedipine clearance had been determined (University of Newcastle upon Tyne, UK); 5 family members, one of whom was a poor nifedipine metabolizer (University of M ichigan); 12 pediatric patients (St. Jude Children's Research Hospital); 14 subjects whose hepatic CYP3A phenotype had been determined by using the erythromycin breath test (University of M ichigan); 10 subjects whose intestinal CYP3A4 content had been determined (University of M ichigan); hepatocytes from 10 peoplefor whom the drug induction of CYP3A protein had been characterized

CYP3AP1 and CYP3A4 genotypes: Caucasians, $\chi^{2}=6.08 ; \mathrm{P}=0.01 ;$ African Americans, $\chi^{2}=12.26 ; \mathrm{P}=0.0004$. CYP3A4 and CYP3A5 genotypes: Caucasians, Fishers exact test $\mathrm{P}=0.058$; African Americans, Fishers exact test $\mathrm{P}=0.28$. 
(INSERM and M edical College of Virginia); 11 pairs of small intestine and liver (some liver samples are the same as those mentioned above) and intestines of 3 Caucasians whose midazolam 1'-hydroxylase and 4-hydroxylase activities and CYP3A5 protein had been characterized (University of Washington). We sequenced the CYP3AP1 promoter and CYP3A5 introns 3,4 and 6 in samples from 10 M exicans, 9 Japanese, 10 Chinese, 10 Southeast Asians, 6 Pacific Islanders and 5 Southwestern American Indians (Human Variation Panels, Coriell Cell Repositories). Informed consent was obtained from all people and approval of this research was obtained according to the relevant institutional guidelines.

Primers and sequencing. We used primer3 (http://www.genome. wi.mit.edu/cgi-bin/primer/primer3.cgi) to design primers to amplify the promoter regions of CYP3A4, CYP3A5, CYP3AP1 and CYP3A7; the amplicons were 400-600 bp. The sequences of the primers were as follows: CYP3A4, 5'-TGGGATGAATTTCAAGTATTTTG-3' (f) and 5'-AGGTTTCCATGGCCAAGTCT-3' $(r)$; CYP3A4 primers to sequence the distal PXRE sequences (nt $-7,836$ to -7,208), 5'-CCGATCAGAATAAGGCATTG- $3^{\prime}(f)$ and $5^{\prime}$-GATTCACCTGGGGTCAACAC-3' $(r)$; CYP3AP1 primers, 5'-GGGGATGGATTTCAAGTATTCTG-3' (f) and 5'-GTCCATCGCCACTTGCCTTCT-3' (r); CYP3A7 primers, 5'-GTCTGGCTGGGTATGAAAGG-3' (f) and 5'-GCCAAGTTTGGGATGAGAT-3' (r); CYP3A5, 5'-GAGGATGGATTTCAATTATTCTA-3' (f) and 5'-GTCCATCGCCACTTTCCTTC $-3^{\prime}(r)$. Forward and reverse primers weretailed with universal sequencing primers (-40 M 13 and $-28 \mathrm{M} 13$, respectively). Primer pairs were used for 35 cycles to amplify genomic DNA. The following conditions were used in each cycle: $95^{\circ} \mathrm{C}$ for $15 \mathrm{~s}, 61^{\circ} \mathrm{C}$ for $30 \mathrm{~s}$, and 72 ${ }^{\circ} \mathrm{C}$ for $5 \mathrm{~min}$. We removed unincorporated nucleotides and primers by incubating the PCR product with shrimp alkaline phosphatase and exonuclease for $30 \mathrm{~min}$ at $37^{\circ} \mathrm{C}$ followed by $15 \mathrm{~min}$ at $80^{\circ} \mathrm{C}$ (ref. 42). Primers for amplification of the full-length CYP3A5 CDNA were P1, 5'-AACAGCCCA GCAAACAGCAGC- $3^{\prime}(\mathrm{f})$, and P2, 5'-TAAGCCCATCTTTATTTCAAGGT $-3^{\prime}(r)$. Primers for sequencing the CYP3A5 CDNA were as follows: P3, 5'-GTTGCTATTAGACTTGAGAGGACT-3'; P4, 5'-TGTAAGGATCTAT GCTGTCCTTC-3'; P5, 5'-CACAAATCGAAGGTCTTTAGGC-3'; P6, 5'-TCAAAAACTGGGGTAAGGAATG-3'; P7, 5'-GCCTAAAGACCTTC GATTTGTG-3'; P8, 5'-CATTCCTTACCCCAGTTTTTGA-3'; P9, 5'-AGT CCTCTCAAGTCTAATAGCAAC-3;; P10, 5'-GAAGGACAGCATAGATC CTTACA-3'; P11, 5'-CAGGGTCTCTGGAAATTTGACA-3'; P12, 5'-TCA TTCTCCACTTAGGGTTCCA-3'; and P13, 5'-CAGCATGGATGTGAT TACTGGC -3 '. The primers used to amplify CYP $3 A 5$ exon $3 B, 4 B$ and $5 B$ insertions from genomic DNA were 5020 22719, 5'-CCTGCCTT CAATTTTTCACTG-3' (f), and 5020_24161, 5'-GCAATGTAGGAAGGAGGGCT- $3^{\prime}(r)$. The nested primers used to sequence the nt 22,893 site were 5020 22743, 5'-TAATATTCTTTTTGATAATG-3' (f), and 5020 23205, $5^{\prime}$-CATTCTTTCACTAGCACTGTTC- $3^{\prime}(r)$. Thenested sequencing primers used to sequence thent 24,035 site were 5020 23761, $5^{\prime}$-CAACAAAAACCG GCAAACTG-3' (f), and 502024135 , 5'-AGGATTTTCAGACTTAA CAC $-3^{\prime}(r)$. The primers used to amplify the exon 7 deletion in CYP3A5*6 were 5020_28814, 5'-GGTCATTGCTGTCTCCAACC-3' (f), and the P6 primer $(r)$, and to sequence across exon 7 5020 30237, 5'-TAT GACTGGGCTCCTTGACC-3' (f), and 5020_30745, 5'-'TGGAATTGTAC CTTTTAAGTGGA-3' $(r)$.

We sequenced the proximal promoters by performing standard fluorescence-based sequencing with Amersham ET Dye Primers. The distal promoter region of CYP3A4 and the CYP3A5 promoter were sequenced by using BigDye Terminator sequencing, and products were resolved by polyacrylamide gel electrophoresis or by capillary gel electrophoresis. The resultant trace files were basecalled by phred and assembled by phrap (http://www.genome.washington.edu). Polyphred ${ }^{42}$ was used to detect potential heterozygosity. To be a true variant, the variant-containing sequence generated by theforward primer had to beidentical to that generated by the reverse primer.

Western-blot analysis. We performed quantitative immunoblotting of CYP3A5 and CYP3A4 content of tissue preparations as described ${ }^{16}$; purified CDNA-expressed CYP3A5 (a gift from R. Peter) and CYP3A4 purified from human liver were the reference standards ${ }^{11}$. Liver microsomes (10-20 $\mu \mathrm{g})$ and intestinal homogenates $(50 \mu \mathrm{g})$, both of which had been prepared from organ donor tissue ${ }^{16}$, were resolved by electrophoresis, and CYP3A5 was detected with anti-CYP3A5 antibody (Gentest) or anti-CYP3A4 anti- body ${ }^{16}$. We determined the integrated optical density of each band by using a ChemiD oc (Biorad) and Quantity One program.

Midazolam kinetic protocol. Midazolam, ${ }^{15} \mathrm{~N}_{3}$-midazolam, 1'-OH M DZ and 4-OH M DZ were provided by Roche Laboratories. All incubations were performed in duplicate in solutions containing potassium phosphate $(0.1 \mathrm{M}, \mathrm{pH} 7.4)$ and human liver microsomes $(20-100 \mu \mathrm{g})$ or intestinal homogenates $(50-100 \mu \mathrm{g})$ or recombinant CYP3A4 or CYP3A5 (10 pmol; Gentest). Midazolam (final concentration, $8 \mu \mathrm{M}$ ) was added to the diluted tissue preparations, and the mixtures were preincubated at $37{ }^{\circ} \mathrm{C}$ for $5 \mathrm{~min}$. We added NADPH (final concentration, $1 \mathrm{mM}$ ) to initiate the reaction. Incubation of human liver microsomes lasted for $2 \mathrm{~min}$; incubation of recombinant CYP3A and intestinal samples lasted for $4 \mathrm{~min}$. Reactions were terminated by the addition of 1 $\mathrm{ml}$ of ice-cold $\mathrm{Na}_{2} \mathrm{CO}_{3}(0.1 \mathrm{M}, \mathrm{pH} \sim 11)$. We used NCl GC-M S to measure the quantities of extracted 1'-OH MDZ and 4-OH MDZ (ref. 16). Reaction velocities and product ratios are presented as mean \pm s.d. Statistical comparisons of mean data for different genotypes were performed by using a 2 -sided, unpaired t-test with unequal variances.

RT-PCR of CYP3A7. Total RNA (5-10 $\mu \mathrm{g})$ from human liver was reversetranscribed according to the manufacturer's instructions (Life Technologies). CYP3A7 cDNA was amplified from first-strand CDNA using oligonucleotides CYP3A7(S), 5'-ATTCCAAGCTATGTTCTTCATCAT-3', and CYP3A7(AS), 5'-AATCTACTTCCCCAGCACTGA-3', under described conditions ${ }^{43}$, except the initial denaturation lasted $5 \mathrm{~min}$, the annealing temperature was $58^{\circ} \mathrm{C}$ and the reaction required 25 cycles. The PCR product was analyzed on agarose gels. Amplification of $28 \mathrm{~S}$ rRNA served as a control for RNA integrity.

GenBank accession numbers. CYP3AP promoter, S74700; CYP3AP1*1 promoter consensus sequence, AF35929. Sequences of CYP3A $5 * 3$ alternatively spliced exons, AF355800, AF 355804, AF355801 and AF355802, and CYP3A5*6, AF355803.

\section{Acknow ledgments}

Wethank E. Green for advice and use of facilities. This work was supported in part by grant GM 60346 and the N IH/N I G M S Pharmacogenetics Research Network and Database (U 01GM 61374, http://pharmgkb.org/) under grant U 01GM (GM 61393) and by grants GM 32165, ES08658, P30 CA21765, and CA51001 from the National Institutes of $\mathrm{Health}$ and by the American Lebanese Syrian Associated Charities (ALSAC).

Received 13 October 2000; accepted 21 February 2001.

1. Lander, E.S. et al. Initial sequencing and analysis of the human genome. Nature 409, 860-921 (2001).

2. Venter, J.C. et al. The sequence of the human genome. Science 291, 1304-1351 (2001).

3. Collins, F.S., Brooks, L.D. \& Chakravarti, A. A DNA polymorphism discovery resource for research on human genetic variation. Genome Res. 8, 1229-1231 (1998).

4. Collins, F.S., Guyer, M.S. \& Chakravarti, A. Variations on a theme: cataloging human DNA sequence variation. Science 278, 1580-1581 (1997).

5. Evans, W.E. \& Relling, M.V. Pharmacogenomics: translating functional genomics into rational therapeutics. Science 286, 487-491 (1999).

6. Rendic, S. \& DiCarlo, F.J. Human cytochrome P450 enzymes: a status report summarizing their reactions, substrates, inducers, and inhibitors. Drug Metab. Rev. 29, 413-580 (1997).

7. Cholerton, S., Daly, A.K. \& Idle, J.R. The role of individual human cytochromes P450 in drug metabolism and clinical response. Trends Pharmacol. Sci. 13, 434-439 (1992).

8. Shimada, T., Yamazake, H., Mimura, M., Inui, Y. \& Guengerich, F.P. Interindividual variations in human liver cytochrome P-450 enzymes involved in the oxidation of drugs, carcinogens and toxic chemicals: Studies with liver microsomes of 30 Japanese and 30 Caucasians. J. Pharmacol. Exp. Ther. 270, 414-423 (1994).

9. Watkins, P.B. Cyclosporine and liver transplantation: will the midazolam test make blood level monitoring obsolete? Hepatology 22, 994-996 (1995)

10. Kivisto, K.T., Koremer, H.K. \& Eichelbaum, M. The role of human cytochrome P450 enzymes in the metabolism of anticancer agents: implications for drug interactions. Br. J. Clin. Pharmacol. 40, 523-530 (1995).

11. Huang, Z., Guengerich, F.P. \& Kaminsky, L.S. 16 $\alpha$-hydroxylation of estrone by human cytochrome P4503A4/5. Carcinogenesis 19, 867-872 (1998).

12. Finta, C. \& Zaphiropoulos, P.G. The human cytochrome P4503A locus. Gene evolution by capture of downstream exons. Gene 260, 13-23 (2000).

13. Schuetz, J.D., Beach, D.L. \& Guzelian, P.S. Selective expression of cytochrome P450 CYP3A mRNAs in embryonic and adult human liver. Pharmacogenetics 4, 11-20 (1994).

14. Wrighton, S.A., Ring, B.J., Watkins, P.B. \& Vandenbranden, M. Identification of a 
polymorphically expressed member of the human cytochrome P-450III family. Mol. Pharmacol. 36, 97-105 (1989).

15. Schuetz, J.D., Molowa, D.T. \& Guzelian, P.S. Characterisation of a cDNA encoding a new member of the glucocorticoid-responsive cytochromes P450 in human liver. Arch. Biochem. Biophys. 274, 355-365 (1989).

16. Paine, M.F. et al. Characterization of interintestinal and intrainestinal variations in human CYP3A-dependent metabolism. J. Pharmacol. Exp. Ther. 283, 1552-1562 (1997).

17. Paulussen, A. et al. Two linked mutations in transcriptional regulatory elements of the CYP3A5 gene constitute the major genetic determinant of polymorphic activity in humans. Pharmacogenetics 10, 415-424 (2000).

18. Lehmann, J.M. et al. The human orphan nuclear receptor PXR is activated by compounds that regulate CYP3A4 gene expression and cause drug interactions J. Clin. Invest. 102, 1-8 (1998).

19. Blumberg, B. et al. SXR, a novel steroid and xenobiotic sensing nuclear receptor Genes Dev. 12, 3195-3205 (1998).

20. Rebbeck, T.R., Jaffe, J.A., Walker, A.H., Wein, A.J. \& Malkowicz, S.B. Modification of clinical presentation of prostate tumors by a novel genetic variant in CYP3A4. J. Natl. Cancer Inst. 90, 1225-1229 (1998).

21. Gonzalez, F.J. The molecular biology of cytochrome P450s. Pharmacol. Rev. 40 243-288 (1989)

22. Sata, F. et al. CYP3A4 allelic variants with amino acid substitutions in exons 7 and 12: evidence for an allelic variant with altered catalytic activity. Clin. Pharmacol. Ther. 67, 48-56 (2000).

23. Goodwin, B.J., Hodgson, E. \& Liddle, C. The orphan human pregnane X receptor mediates the transcriptional activation of CYP3A4 by rifampicin through a distal enhancer module. Mol. Pharmacol. 56, 1329-1339 (1999).

24. Aoyama, T. et al. Cytochrome P-450 hPCN3, a novel cytochrome P-450 IIIA gene product that is differentially expressed in adult human liver. J. Biol. Chem. 264, 10388-10395 (1989).

25. Maquat, L.E. When cells stop making sense: effects of nonsense codons on mRNA metabolism in vertebrate cells. RNA 1, 453-465 (1995)

26. Maquat, L.E. The power of point mutations. Nature Genet. 27, 5 (2001).

27. Jounaidi, Y., Hyrailles, V., Gervot, L. \& Maurel, P. Detection of a CYP3A5 allelic variant: a candidate for the polymorphic expression of the protein? Biochem Biophys. Res. Commun. 221, 466-470 (1996).

28. Gorski, J.C., Hall, S.D., Jones, D.R., BandenBranden, M. \& Wrighton, S.A Regioselective biotransformation of midazolam by members of the human cytochrome P4503A (CYP3A) subfamily. Biochem. Pharm. 47, 1643-1653 (1994).

29. Felix, C.A. et al. Association of CYP3A4 genotype with treatment-related leukemia. Proc. Natl. Acad. Sci. USA 95, 13176-13181 (1998).
30. Paris, P.L. et al. Association between a CYP3A4 genetic variant and clinical presentation in African-American prostate cancer patients. Cancer Epidemiol. Biomarkers Prev. 8, 901-905 (1999).

31. Westlind, A L L \& Interindividual differences in hepatic expression of CYP3A4: Relationship to genetic polymorphism in the 5 -upstream regulatory region. Biochem. Biophys. Res. Commun. 259, 201-205 (1999).

32. Ball, S.E. et al. Population disribution and effects on drug metabolism of a genetic variant in the 5 ' promoter region of CYP3A4. Clin. Pharmacol. Ther. 66, 288-294 (1999).

33. Hashimoto, H. et al. Gene structure of CYP3A4, an adult-specific form of cytochrome P450 in human livers, and its transcriptional control. Eur. J. Biochem. 218, 585-595 (1993).

34. Cooper, T.A. \& Mattox, W. The regulation of splice-site selection, and its role in human disease. Am. J. Hum. Genet. 61, 259-266 (1997).

35. Wrighton, S.A. et al. Studies on the expression and metabolic capabilities of human liver cytochrome P450IIIA5 (HLp3). Mol. Pharmacol. 38, 207-213 (1990)

36. Gillam, E.M.J. et al. Expression of cytochrome P450 3A5 in Escherichia coli: effects of 5' modification, purification, spectral characterization, reconstitution conditions, and catalytic activities. Arch. Biochem. Biophys. 317, 374-384 (1995).

37. Grogan, W.M., Phillips, V.M., Schuetz, E.G., Guzelian, P.S. \& Watlington, C.O. Corticosterone 6b-hydroxylase in A6 epithelia: a steroid-inducible cytochrome P450. Am. J. Physiol. Cell Physiol. 258, C480-C488 (1990).

38. Schuetz, E.G. et al. Expression of cytochrome P4503A in amphibian, rat and human kidney. Arch. Biochem. Biophys. 294, 206-214 (1992).

39. Haehner, B.D. et al. Bimodal distribution of renal cytochrome P450 3A activity in humans. Mol. Pharmacol. 50, 52-59 (1996).

40. Halushka, M.K. et al. Patterns of single-nucleotide polymorphisms in candidate genes for blood-pressure homeostasis. Nature Genet. 22, 239-247 (1999)

41. Cargill, M. et al. Characterization of single-nucleotide polymorphisms in coding regions of human genes. Nature Genet 22, 231-238 (1999).

42. Nickerson, D.A., Tobe, V.O. \& Taylor, S.L. PolyPhred: automating the detection and genotyping of single nucleotide substitutions using fluorescence-based resequencing. Nucleic Acids Res. 25, 2745-2751 (1997).

43. Schuetz, E.G. Beck, W.T \& Schuetz, J.D. Modulators and substrates of Pglycoprotein and cytochrome P4503A coordinately up-regulate these proteins in human colon carcinoma cells. Mol. Pharmacol. 49, 311-318 (1996).

44. Nebert, D.W. Suggestions for the nomenclature of human alleles: relevance to ecogenetics, pharmacogenetics and molecular epidemiology. Pharmacogenetics 10, 279-290 (2000) 